\title{
Surgical Management of Oral Submucous Fibrosis Defects Using Buccal Fat Pad in Posterior Region and Nasolabial Flap in The Anterior Region
}

Pavan Kumar B ${ }^{1}$, Brahmaji Rao J 2, Haripriya Chari³, J eevan Kumar KA ${ }^{4}$, Madhusudan Rao $\mathrm{G}^{5}$

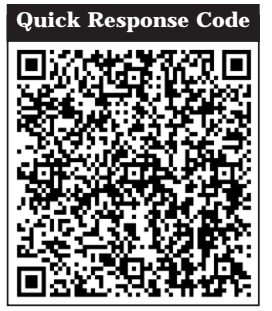

doi: $10.5866 / 2016.8 .10082$

${ }^{1}$ Professor \& HOD

${ }^{2,4}$ Professor,

${ }^{3}$ Senior Lecturer

${ }^{5}$ Reader,

Department of Oral and Maxillofacial Surgery, Kamineni Institute of Dental Sciences,

Narketpally

\section{Article Info:}

Received: April 10, 2016

Review Completed: May 11, 2016

Accepted: J une 13, 2016

Available Online: September, 2016 (www.nacd.in)

(c) NAD, 2016 - All rights reserved

\section{Email for correspondence:}

pavankumarbatchu40@gmail.com

\begin{abstract}
:
Background: Oral submucous fibrosis is a chronic debilitating disease characterized by gradually increasing fibrosis of the oral cavity and pharynx, mainly the buccal mucosa, resulting in trismus. Various flaps have been used to reconstruct the surgical defects
\end{abstract} following excision of fibrous bands.

Aim: The aim of the present study was to clinically evaluate the application of pedicled buccal fat pad (BFP) and nasolabial flap (NLF) in the surgical management of stage III and IV oral submucous fibrosis (OSMF).

Materials and Methods: Twenty cases of OSMF of clinical stage III and stage IV were selected. All the patients underwent incision of fibrotic bands and coverage of the buccal defect anteriorly with a nasolabial flap and posteriorly with a pedicled BFP flap. All patients were analyzed for mouth opening, time taken for epithelialization, cheek flexibility, scar formation and intercommissural width.

Results: The study showed epithelization for BFP took an average time of 4 weeks and mucosalization of NLF took a mean time of 5months. Post operative mouth opening and intercommissural width were increased significantly and minimal scar formation was observed.

Condusion: It can be conduded that the results achieved are better with use of both NLF and BFP simultaneously rather than any of these flaps used alone. Amount of the defect that could be satisfactorily closed by the use of NLF along with BFP is greater with minimal tension when compared with the NLF or BFP alone. Widening of oral commissure is less when compared with NLF alone.

Key words: oral submucous fibrosis, nasolabial flap, buccal fat pad

\section{INTRODUCTION:}

Oral Sub-mucous Fibrosis is a chronic debilitating disease of the oral cavity characterized by inflammation and progressive fibrosis of the sub mucosal tissues. ${ }^{1}$ The clinical features of OSMF includes progressive inability to open the mouth (Trismus) due to oral fibrosis and scarring, pain and burning sensation upon consumption of spicy food stuffs, increased salivation, change of gustatory 
sensation, hearing loss due to stenosis of the eustachian tubes, dryness of the mouth, nasal tone to the voice, dysphagia (if esophagus is involved). ${ }^{2}$ The mainstay in the treatment of OSMF, is therefore concentrated on attempts to improve the mouth opening. ${ }^{3}$ Several medical and surgical therapies have been put forth with varied degree of success. Amongst the most widely accepted and frequently used surgical procedures are use of buccal fat pad or nasolabial flaps. ${ }^{4-7}$ In the experience of majority of surgeons it has been found that the rotation of nasolabial flaps in to the oral cavity and suturing these flaps in to the posterior most aspect of the defect is very difficult, in addition the difficulty is coupled by poor access and visibility in this area. These findings have prompted us to take up the present study of closure of the surgical defects in oral sub mucous fibrosis, posteriorly by buccal fat pad and anteriorly by nasolabial flaps. Till now there are studies either only on buccal fat pad or on nasolabial flaps.

\section{MATERIALS AND METHODS:}

A total of twenty patients were selected with oral submucous fibrosis, staged III and IV according to Khanna and Andrade classification. Patients who were willing to quit the habits were included in the study. The exclusion criteria were patients with stage I and stage II oral sub-mucous fibrosis, below 18 years of age, uncontrolled systemic conditions and TMJ problems.

\section{Surgical method:}

After informed consent the operation was performed under general anesthesia with nasal intubation. Incision was made from the angle of the mouth to the anterior tonsillar pillars down to the muscles, depending upon the location of the fibrotic bands (Figure 1) which restricted mouth opening (Figure 2). The mouth was forcefully open with a mouth opener to an acceptable range of approximately $35 \mathrm{~mm}$. If intraoperative mouth opening is less than $35 \mathrm{~mm}$, then bilateral coronoidectomy or temporalis myotomy was done through the same incision. The buccal defects were then reconstructed by NasoLabial Flap anteriorly (FIG. 3, 4 and 5) and Buccal Fat Pad posteriorly(Figure 6). All the patients werefollowed up for 6 months.

The following parameters were assessed. Duration of surgical procedure was measured from starting of the operative procedure till the surgical defect is closed. Mouth opening was assessed pre operatively and post operatively (Figure 7). Cheek flexibility was assessed clinically and areas with more suppleness were given ++ and areas where there was feeling of fibrous scar tissue or hardness of mucosa were given + . Inter commissural width was assessed using a scale and the distance between corners of the mouth on both the sides was measured. Time taken for the transposed nasolabial flaps to be fully covered with mucosa and complete epithelization of buccal fal pad was assessed through regular follow up. The esthetic outcome of the scar was assessed by observing the color and character of thescar and a score was given accordingly (F igure 8).

\section{CHARACTER OF THE SCAR}

01 - Noperceptible scar

02 - Visible but thin and linear scar

03 - Wide scar

04 - Hypertrophic scar or keloid

\section{COLOR OF THE SCAR}

$$
\begin{aligned}
& 01 \text { - N operceptible scar } \\
& 02 \text { - Normal skin color } \\
& 03 \text { - Red } \\
& 04 \text { - Hyperpigmented } \\
& 05 \text { - Hypopigmented }
\end{aligned}
$$

\section{RESULTS:}

A total of 20 patients were operated with ages ranging from 25-66 years, with a pre-operative mouth opening from 6-17 mm (mean - $12.7 \mathrm{~mm}$ ). Patients were assessed for mouth opening in the immediate post op, 15 days, 1st month, 3rd month and 6 th month. The mean duration for epithelization of buccal fat pad was 4 weeks and for mucosilization of nasolabial flap was 5.65 months. Widening of the oral commissure was seen post surgery. The pre operative inter commissural distance ranged from 45-65 mm (mean 58.4mm ) while the six months post operative values ranged from 49-69 mm (mean $62.4 \mathrm{~mm}$ ) with difference in the pre operative and post operative values ranging from 3-5 mm (mean of $4 \mathrm{~mm}$ ).

Operating time was assessed for all the 20 patients and a mean operating time of $2 \mathrm{hr} 30.3 \mathrm{~min}$ (150.3 min) was recorded. 


\section{DISCUSSION:}

Pindborg has defined Oral Submucous Fibrosis (OSMF) as "an insidious chronic disease affecting any part of the oral cavity and occasionally extending to the pharynx and esophagus, although, occasionally preceded by and/or associated vesicle formation. It is always associated with juxtaepithelial inflammatory reaction followed by fibroelastic changes in the lamina propria, with epithelial atrophy leading to stiffness of the oral mucosa causing trismus and difficulty in eating". ${ }^{8}$

The common sites involved are labial mucosa, buccal mucosa, retromolar pads, soft palateand floor of the mouth. Fibrotic changes of the pharynx, esophagus and paratubal muscles of eustachian tubes had been reported. The fibrotic bands in the buccal mucosa run in vertical directions, in the soft palate the fibrous bands radiate from the pterygomandibular raphe or the anterior faucial pillars and in the lips circular bands can be felt around entire rima oris. ${ }^{9}$ The uvula is markedly involved in the late stages which shrinks and appears like a small bud. ${ }^{10}$ Patients complain of burning sensation, nasal regurgitation or nasal intonation and restricted mouth opening. It is associated with the betel nut, betel quid, tobacco and pan (a betel leaf folded around a mixture of tobacco, betel nut, lime, catechu and other flavouring agents) chewing habits including frequency (number of times per day), duration (years of consumption) and type (Gutkha, pan masala, Mava etc) was recorded. The areca nut (Areca catechu) is considered as the main etiologic factor causing OSMF, it generates free radicals and causes local immunosuppression. Seedat and Van Wyk in 1988 reported that OSMF also occurs in patients having no history of areca nut chewing. ${ }^{11-13}$ F urthermore it is reported that $7-13 \%$ of cases of OSF transform into squamous cell carcinoma according to Tilakaratne et al in 2006, although none of the patients in the present study have transformed into oral cancer. In the present study all the patients had a history of areca nut consumption for more than 8-10 years. ${ }^{14}$

Khanna J $\mathrm{N}$ and Andrade NN devel oped a group classification system for the surgical management of OSMF. ${ }^{15}$

Group I: Very early cases: Common symptom is burning sensation in the mouth, acute ulceration, recurrent stomatitis and not associated with mouth opening limitation.
Group II: Early cases: Buccal mucosa appears mottled and marble like, widespread sheets of fibrosis palpable, interincisal distance of 26 to 35 $\mathrm{mm}$.

Group III: Moderately advanced cases: Trismus, interincisal distance of 15 to $25 \mathrm{~mm}$, buccal mucosa appears pale, firmly attached to underlying tissues, atrophy of vermilion border, vertical fibrous bands palpable at the soft palate, pterygomandibular raphe and anterior faucial pillars.

Group IVA: Advanced cases: Severetrismus, interincisal distance of less than $15 \mathrm{~mm}$, thickened faucial pillars, shrunken uvula, restricted tongue movement, presence of circular band around entire lip and mouth.

Group IVB: Advanced cases with presence of hyperkeratotic leukoplakia and/or squamous cell carcinoma.

The ideal goals of therapy include amelioration of the symptoms (burning sensation, restriction of mouth opening), and preventing further disease progression and malignant transformation. Various treatment modalities like medical, surgical are available to improve the patient condition. The medical management includes sub mucosal injection of steroids, placental extracts, hyaluronidase. This may give temporary relief but aggravates fibrosis because of the multiple needle insertion and irritation from the drug. The medical management is palliative therapy which is not going to reverse the condition completely. Surgical excision of bands is a preferred treatment procedure followed by reconstruction of the soft tissue defects after resection of fibrous bands.

In the present study, we considered Group III and IV for surgical treatment and Group I and II for medical treatment. The surgical aspect includes excision of OSMF bands followed by reconstruction with single stage bilateral nasolabial flap, which is covering the raw area created by excision of cheek mucosa anteriorly, and reconstruction with and buccal pad of fat posteriorly followed by supportive therapy with antioxidants.

In the present series of 20 , all patients gave a positive history of chewing some form of betel nut or tobacco or a combination of the common form being roasted betel nuts. They had chief complaint of burning sensation upon eating spicy food, hot food 


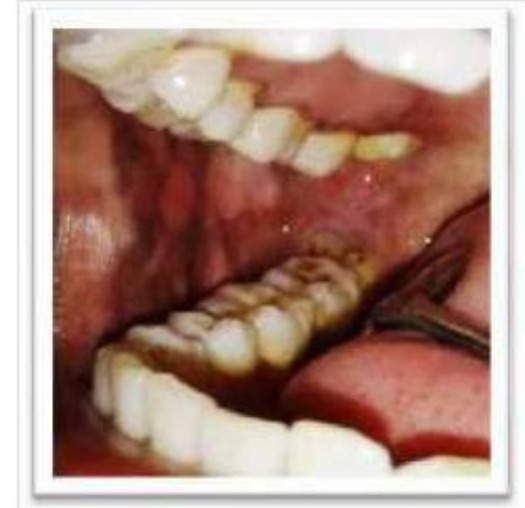

Figure 1: Preoperative picture showing fibrous bands

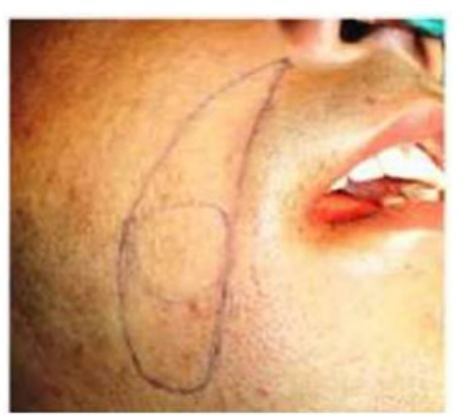

Figure 3: Naso labial flap marked

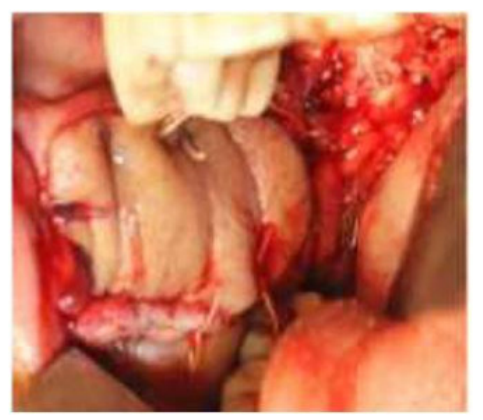

Figure 5: Nasolabial flap covering anterior region after excision

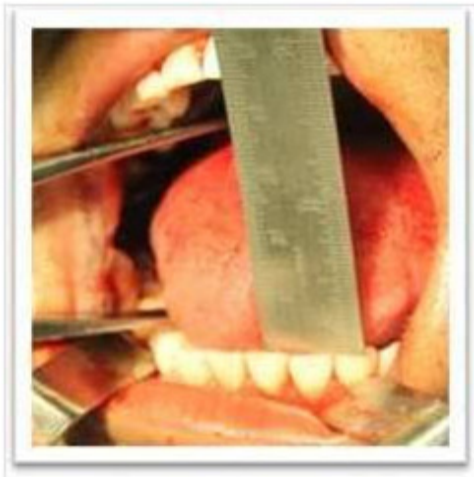

Figure 7: Post operative picture showing improved mouth opening

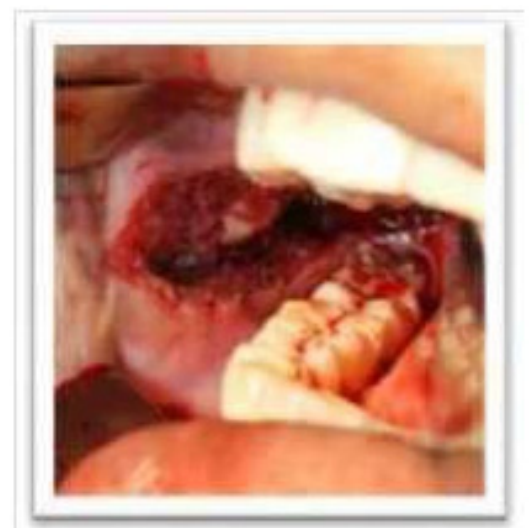

Figure 2: Intra-operative band excision

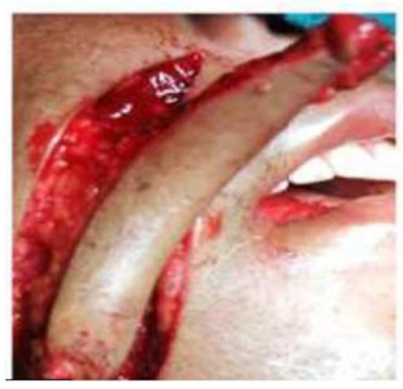

Figure 4: Nasolabial flap rotated intra orally

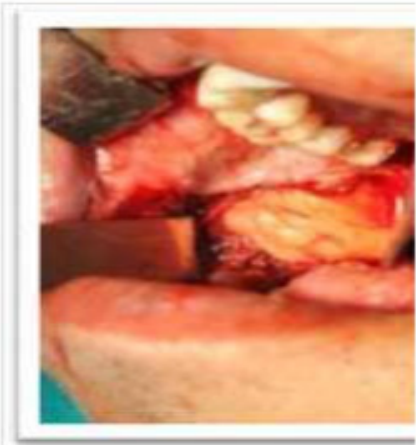

Figure 6: Buccal fat pad in the posterior region

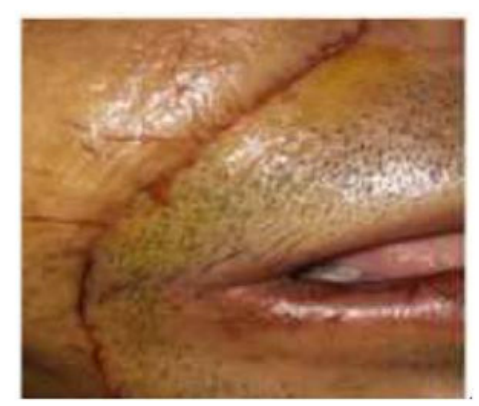

Figure 8: Post operative picture showing minimal scar in nasolabial region

Indian J Dent Adv 2016; 8(2): 82-88 
or on intake of hot beverages and none of them complained of nasal regurgitation or nasal intonation. According to Khanna and Andrade's ${ }^{14}$ grouping of OSMF based on clinical and histolopathogic features, 03 were group III while 17 belonged to group IVa.

All the patients were counseled pre-operatively regarding the treatment outcomes and quitting of the habit to avoid any chances of late post-surgical relapse. In all cases it was ensured that the patients stopped the habit at least three months prior surgery. They were given multivitamin therapy with antioxidants prior to surgery and continued even after the surgery at least for 6 months. Post operatively, all patients were advised to take bland diet

Muhammad et al also recommended coronoidectomy in cases of trismus to achieve excellent mouth opening. All the patients in this study had coronoidectomy or temporalis myotomy as a routine procedure if the achieved mouth opening was less than $35 \mathrm{~mm}$ after excision of fibrous bands.

R.M. Borle et al has extensively used the extended NLF in the management of OSMF. Extended NLF is raised from the tip of NLF to the inferior border of mandible in the plane of the superficial musculoaponeurotic system from both terminal points to the region of the central pedicle. The pedicle is $1 \mathrm{~cm}$ lateral to the corner of mouth and the diameter of the pedicle is roughly $1 \mathrm{~cm}$. The flap is transposed intraorally through a small transbuccal tunnel near the commissure of the mouth with no tension and sutured over intraoral defect.

TheBF P was first identified by Heister in 1727. Egyedi (1977) first described the use of the BFP for closure of persistent oro-nasal or oro-antral communications. ${ }^{16}$ BFP harvested through an intraoral surgical site is simple, easy and has no donor site morbidity. On an average, the volume of BFP is $9.6 \mathrm{~mL}$ (range, 8.3-11.9 mL). Defects measuring up to 3-5 centimeters $(\mathrm{cm})$ can be covered with BFP without compromising the blood supply was concluded in a study conducted by Stuzln et al. ${ }^{17}$

The present study was aimed at using both these versatile flaps in combination to reconstruct the defects following excision of fibrous bands in oral submucous fibrosis. In many occasions the authors have felt the approximation of the nasolabial flaps in retromolar trigone region or beyond is very difficult due to limited access. This technique is also very time consuming. Since we have to cover a large area of the defect, a large nasolabial flap needs to be raised which again leads to widening of the commissure. Since the versatility and usefulness of the buccal fat pad was already proved and particularly due to its proximity to posterior region, raising of the buccal fat pad and covering of the remaining defect with nasolabial flap raised on a small area, will reduce the efforts of the surgeon in reaching inaccessible areas also reducing the range of widening of commissure.

In our study, the age range was 25 to 60 years, and 11 were male and 9 were female, suggestive of high prevalence rates in males which is similar to male predominance, reported by various authors.

All the patients presented with a range of pre operative mouth opening from 6 tol7 mm (mean $12.7 \mathrm{~mm}$ ). Patients were assessed for six months post operatively. Post operatively all the cases had a mean mouth opening of $43.95 \mathrm{~mm}$ ranging from 35 to $48 \mathrm{~mm}$. Borle et al observed that interincisal mouth opening improved significantly from a mean of $14 \mathrm{~mm}$ (range $3-23$ ) to a mean of $41 \mathrm{~mm}$ (range 23 55) using NLF. ${ }^{7}$ Sharma et al also used BFP for reconstruction of the defect in Stage III and IV OSMF patients. ${ }^{18}$ The mouth opening after 1-year follow-up was $35 \mathrm{~mm}$ in stage III group and 31.76 $\mathrm{mm}$ in stage IV group. Vigorous postoperative physiotherapy was necessary to maintain the postoperative mouth opening achieved intraoperatively.

In the present study, epithelization of BFP was seen with a mean of 4 weeks duration. It was 3 weeks in cases - 1, 5, 7, 10, 15 and 18; 4 weeks in cases - 3, 4, 6, 8, 9, 11, 14, 16 and 19; 5 weeks in cases - 12, 13 and 20; 6 weeks in case no - 17. However Tideman et al first described the use of the uncovered BF $P$ as a pedicled graft in the mouth, and showed that epithelization occurred within 2-3 weeks, leaving a good mucosal surface. ${ }^{19}$

Donor site morbidity is negligible with the use of NLF. It effectively covers both posterior and anterior defect of fibrotomy in cases of OSMF. Extraoral scar, infection, minor or major flap necrosis, wound dehiscence do occur in a small number of patients as stated by Ioannides and 
Fossion. ${ }^{20}$ Asymmetry at the level of the nasolabial fold is noted if proper suturing is not done. The most common complication with the use of NLF was the extraoral scar and intraoral growth of hairs over the flap. Garatea et al recommended the use of a cheek rotation flap for aesthetic postoperative scar . ${ }^{21}$ In the present study the post operative extraoral scars were hidden in the NLF. Out of the 20 patients, in 2 cases the character of the scar was not perceptible. In 16 cases thin and linear scar was visible. In two cases scar is wide but acceptable. The color of the scar merged with normal skin color in 18 cases and not perceptible in 2 cases. Intra oral hair growth was seen in all the male patients that reduced significantly after 6 months post operatively. The preoperative mean inter commissural distance was $58.4 \mathrm{~mm}$ which extended to $62.4 \mathrm{~mm}$ after 6 months postoperatively, with an increase by $4.00 \mathrm{~mm}$. The mean duration of surgery was 2 hours 30 minutes which was less when compared with the use of NLF alone. The combined technique has considerably reduced operating time. This was due to the ease of access and ease of adaptation of the BFP to the posterior aspect of the defect.

Possible intra operative complications included damage to facial vessels, stenson's duct and branches of facial nerve. However we have not encountered these in any of the 20 cases. The extent of the defect that was replaced by NLF along with BFP was much wider with minimal tension in comparison with buccal fat pad or nasolabial flap alone. The size of the defect that can be covered by buccal fat pad was on an average of $3-5 \mathrm{~cm}$ and nasolabial flap can cover the defect upto $9 \mathrm{~cm}$. Though nasolabial flap can cover a large area up to anterior faucial pillars, adaptation of this flap to this area is difficult and in the experience of many surgeons, the posterior teeth ( $1^{\text {st }}$ and $2^{\text {nd }}$ molars) were sacrificed to facilitate its suturing in posterior most aspect. By combining both the flaps in this study, the suturing of buccal fat pad was accessible and easy in posterior region. So, this procedure facilitates easy suturing eliminating the need for extracting the posterior teeth.

Regarding cheek flexibility, the cheeks were soft and elastic in areas covered by nasolabial flap 6 months postoperatively where as in areas covered by buccal fat pad it was slight fibrous and less elastic. But this did not hamper the inter incisal opening.

\section{THE HEALING PATTERNS OF THE FLAPS WERE AS FOLLOWS:-}

HEALING OF NASOLABIAL FLAP: During 1st Week- Close apposition of the edges of flap and the oral mucosa. All the sutures were intact without breaking down of wound, 1st month - Better fusion of edges, 3rd month - The graft intra oral site predominantly seen still in the form of keratinized epithelium with minute pinkish areas, 4th month The grafted intra oral site shows mixed appearance of pinkish mucosa like areas mixed with keratinized mucosa, 6th month - Minor patchy areas of keratinized epithelium seen with no differentiation of the borders of the flap with the mucosa and a predominantly well healed pinkish mucosa like areas were seen.

HEALING OF BUCCAL FAT PAD: During 1st Week - loose apposition of the flap with the edges of the oral mucosa. Hence vigorous oral prophylaxis and irrigations were avoi ded during this period, 2nd Week - raw areas of the defect covered by the grafted flap. Minor sloughing of the graft superficially observed, 3rd Week - the grafted flap appeared healthier, gaining pinkish colour both from center and edges. At this stage all unresorbed sutures were removed, 4th Week- Complete epithelization of the fat was nearly complete, the fat was blended with normal mucosa with softening and elasticity of the transposed mucosa improved.

\section{CONCLUSION}

The advantages we have found while using the NLF and BFP together are that it was a relatively easy procedure with proximity of the donor sites, rich vascular supply of the flaps, versatility in design, easy flap elevation and causing minimal esthetic deformity. Amount of the defect that could be satisfactorily closed by the use of NLF along with BFP is greater with minimal tension when compared with the NLF or BFP alone. Widening of oral commissure is less when compared with NLF alone. Scar perception is less when compared with NLF alone. There is reduced operating time with less intraoral scar retraction when compared with BFP alone, so more chances of maintaining the achieved mouth opening. 


\section{REFERENCES:}

1. Gnanam A, Kannadasan K, Venkatachalapathy S, David $J$. Multimodal treatment options for oral submucous fibrosis. SRM Univ J Dent Sci 2010; 1(1):26-29.

2. N Dyavanagoudar S. Oral Submucous Fibrosis: Review on Etiopathogenesis. J Can Sci Therapy 2009; 1(2):72-77.

3. Agarwal R, Kaushal A, Singh R, U padhyay Y. Management of oral submucous fibrosis by different surgical approaches: report of three cases. Case Reports 2013.

4. Mehrotra D, Pradhan R, Gupta S. Retrospective comparison of surgical treatment modalities in 100 patients with oral submucous fibrosis. Oral Surg Oral Med Oral Path Oral Rad End 2009; 107(3):1-10.

5. Yeh C. Application of the buccal fat pad to the surgical treatment of oral submucous fibrosis. Int J Oral Max Surg 1996; 25(2):130-133.

6. Chao C, Chang L, Liu S, Wang J. Histologic examination of pedicled buccal fat pad graft in oral submucous fibrosis. J Oral Max Surg 2002; 60(10):1131-1134.

7. Borle R, Nimonkar P, Rajan R. Extended nasolabial flaps in the management of oral submucous fibrosis. Brit J Oral Maxillofac Surg 2009; 47(5):382-385.

8. Pindborg J J, Sirsat SM. Oral submucous fibrosis. Oral Surg Oral Med Oral Pathol. 1966.

9. Borle R, Borle S. Management of oral submucous fibrosis: A conservative approach. J Oral Max Surg 1991; 49(8):788791.

10. Cox S, Walker D. Oral submucous fibrosis. A review. Aus Dent J 1996; 41(5):294-299.

11. Arakeri G, Brennan PA. Oral submucous fibrosis: an overview of the aetiology, pathogenesis, classification, and principles of management. BrJ Oral Maxill ofac Surg 2013; 51(7):587-593.
12. Mehta A, Panwar S, Verma R, Pal A. Buccal fat pad reconstruction in oral submucosal fibrosis. Med J Armed F orces Ind 2003; 59(4):340-341.

13. Kamath V. Surgical Interventions in Oral Submucous Fibrosis: A Systematic Analysis of the Literature. J Maxill ofac Oral Surg 2014; 14(3):521-531.

14. Tilakaratne W, Klinikowski M, Saku T, Peters T, Warnakulasuriya S. Oral submucous fibrosis: Review on aetiology and pathogenesis. Oral Oncol 2006; 42(6):561-568.

15. Khanna J N, Andrade NN. Oral submucous fibrosis: a new concept in surgical management. Report of 100 cases. Int J Oral Maxillofac Surg 1995; 24(6):433-439.

16. Rai A, Datarkar A, Rai M. Is buccal fat pad a better option than nasolabial flap for reconstruction of intraoral defects after surgical release of fibrous bands in patients with oral submucous fibrosis? A pilot study: A protocol for the management of oral submucous fibrosis. J CranioMaxillofacial Surg 2014; 42(5):111-116.

17. Pers M. Cheek flaps in partial rhinoplasty. Stand J Plast Reconstr Surg 1967; 1:37.

18. Sharma R, Thapliyal GK, Sinha R, Menon PS. Use of buccal fat pad for treatment of oral submucous fibrosis. J Oral Maxillofac Surg 2012; 70(1):228-232.

19. Tideman $\mathrm{H}$, Bosanquet $\mathrm{A}$, Scott $\mathrm{J}$. U se of the buccal fat pad as a pedicled graft. J Oral Maxillofac Surg 1986; 44(6):435440.

20. Ioannides C, Fossion E. Nasolabial flap for the reconstruction of defects of the floor of the mouth. Int J Oral Maxillofac Surg 1991; 20(1):40-43.

21. Garatea J, Buenechea R, Bescos C, Gonzalez E, Bassas C. Intraoral reconstruction with the nasolabial island flap. A modified technique. J Craniomaxillofac Surg 1991; 19(3):119-122.

\section{Gain quick access to our journal online View our journal at www.nacd.in}

